\title{
Twentieth century North Atlantic jet variability
}

\author{
Tim Woollings, ${ }^{\text {a* }}$ Camelia Czuchnicki ${ }^{\mathrm{a}}$ and Christian Franzke ${ }^{\mathrm{b}}$ \\ ${ }^{a}$ Department of Meteorology, University of Reading \\ ${ }^{\mathrm{b}}$ British Antarctic Survey, Cambridge, UK \\ ${ }^{*}$ Correspondence to: Department of Meteorology, University of Reading, Earley Gate, PO Box 243, Reading, RG6 6BB, \\ UK. E-mail: t.j.woollings@ reading.ac.uk
}

Long records of the latitude and speed of the North Atlantic eddy-driven jet stream since 1871 are presented from the newly available Twentieth Century reanalysis. These jet variations underlie the variability associated with patterns such as the North Atlantic Oscillation and have considerable societal impact through variations in the prevailing westerly winds. The ensemble information available in the reanalysis is used to assess the uncertainty in the jet indices, which is found to be relatively small even in the early years of the records. Several aspects of the records are investigated, including the seasonal cycles and spectral characteristics. A key feature is the high variability on sub-seasonal timescales, which is large compared to the seasonal cycle. In general, the features exhibited in shorter records are shown to be robust, for example the multimodality of the jet latitude distribution which suggests the existence of preferred positions of the jet. Decadal variations in jet latitude are shown to correspond to changes in the occurrence of these preferred positions. When viewed in this longer term context the variations of recent decades do not appear unusual, and recent events are not unprecedented in the historical record.

Copyright (c) 0000 Royal Meteorological Society

Key Words: iList up to eight key wordsi

Received...

Citation: ... . 


\section{Introduction}

The midlatitude eddy-driven jet streams are manifested at the surface as the prevailing westerly winds, and as such their variability has considerable societal impact. In the North Atlantic the eddy-driven jet is particularly variable on all timescales from weeks to decades, and this variability is partly described by patterns such as the North Atlantic Oscillation (NAO), Northern Annular Mode (NAM) and East Atlantic pattern (EA). Over recent decades the jet variations described by the NAO/NAM in particular have driven anomalous patterns of temperature, precipitation and storm activity around the Atlantic basin and beyond (Hurrell 1995; Thompson and Wallace 2001; Hurrell and Deser 2009). The change from extreme negative to positive NAO values between the 1960s and 1990s continues to inspire much discussion despite the more recent return to moderate and negative values (Cohen and Barlow 2005; Cattiaux et al. 2010).

North Atlantic variability has been extensively described over the second half of the 20th century using reanalysis products which assimilate both surface and upper air observations. Prior to this, some information on NAO variations, for example, has been inferred from surface pressure reconstructions or station data (Jones et al. 1997; Luterbacher et al. 2001; Pinto and Raible 2012). In a recent advance the Twentieth Century reanalysis project (Compo et al. 2011) assimilated only surface observations in deriving dynamically consistent threedimensional fields back to the late 19th century. This provides an opportunity to analyse the characteristics of jet variability in an observationally-constrained dataset of unprecedented length. For the purpose of evaluating climate models it is useful to determine whether jet characteristics derived from the recent decades are robust features of a longer record.

In this paper we analyse the variability of the latitude and speed of the North Atlantic eddy-driven jet stream in the Twentieth Century reanalysis. These quantities have been derived from the near-surface zonal wind field following the method of Woollings et al. (2010) and can be related in particular to changes in the NAO and EA (Woollings et al. 2010; Woollings and Blackburn 2012). Some studies have diagnosed jet variations and trends in the recent reanalysis period (Strong and Davis 2007; Strong and Davis 2008; Archer and Caldeira 2008; Franzke and Woollings 2011) and one of the key aims of this study is to put these in the context of a longer observational record. A more general aim is to assess the level of jet variability on decadal timescales. One previously unexplored aspect of this is whether the level of intraseasonal jet variability itself exhibits long-term changes in magnitude. This question was inspired by the recent suggestion of Häkkinen et al. (2011) of multi-decadal variations in the frequency of Atlantic/European blocking. We also take this opportunity to describe the seasonal cycles of the jet quantities and their variations between different periods.

\section{Methodology}

Woollings et al. (2010) used zonal wind averaged over 925$700 \mathrm{hPa}$ to derive daily indices of jet latitude and speed. On testing it was found that almost identical results were obtained using only the $850 \mathrm{hPa}$ values (Czuchnicki 2011), so only this level was used for simplicity and to reduce data requirements. The method consists of averaging the zonal wind over the sector $0-60^{\circ} \mathrm{W}$ in the North Atlantic, then applying a 10-day low-pass filter before simply identifying the maximum westerly wind speed within the range 15$75^{\circ} \mathrm{N}$. The location and size of this value are used to define the daily jet latitude and speed respectively. In contrast to Woollings et al. (2010) we do not remove the seasonal cycles of the jet indices; these are instead retained for analysis. Daily data from the Twentieth Century reanalysis of Compo et al. (2011) has been used for the period February 1871 to January 2008, which gives a sample size of 136 full winter seasons and 137 of each of the other seasons. 
One of the novel aspects of the Twentieth Century reanalysis is the use of an ensemble method to provide information on the uncertainty. In regions and periods of sparse observational coverage the model is insufficiently constrained by the observations, resulting in spread between the ensemble members. Here we use this ensemble information to provide uncertainties in the jet diagnostics, simply by repeating the analysis for each of the 56 ensemble members and presenting ranges of each diagnostic across the ensemble.

\section{A cautionary example}

In this paper we focus exclusively on the North Atlantic, partly because of its dynamical significance but also because of the relatively good observational coverage. Similar analyses have been performed for other regions (Czuchnicki 2011) but in these cases the conclusions are limited due to ensemble spread and the associated uncertainty in the early period of the reanalysis. In this section we show one example of these analyses which illustrates that particular care needs to be taken over apparent trends exhibited in this early period.

Figure 1a shows the time series of summertime intraseasonal variability of jet latitude in the eastern North Pacific. The intraseasonal variability is defined here as the standard deviation of the daily jet indices in each 90-day season. In the Pacific case there is an apparent long-term trend of decreasing intraseasonal variability. However, much of this trend occurs in the period before 1940 when the large ensemble spread reflects insufficient observational coverage to constrain the model. It is likely that this trend in fact reflects a drift of the model back towards its unconstrained state which seems to be biased towards overly variable jet speeds. The use of the ensemble information has helped to identify this problem and this shows that misleading results could be obtained when only the ensemble mean is used.

An example of the same diagnostic is given for the North Atlantic in Figure 1b. In this case the range between ensemble members is much smaller, so that the existence of decadal-scale variations in the level of variability can be identified with reasonable confidence. (These are in fact the variations which we focus on in section 7). However, although the spread is much smaller the possibility of this effect contributing to some early variations should not be ruled out.

\section{Spectral characteristics and seasonal cycles of the North Atlantic jet}

Figure 2 shows spectra of the daily jet latitude and speed indices. The spectrum of jet latitude in Figure 2a is generally similar to the theoretical spectrum of the corresponding red noise process (shown by the black line). There is, however, a clear peak at a period of 365 days indicating the presence of a seasonal cycle. The spectrum of jet speed in Figure $2 b$ is less similar to the corresponding red noise process, with less power at periods shorter than 10 days and periods longer than 100 days. This contrast may arise from the nature of the jet definitions, in which the latitude in particular can change rapidly between one day and the next as one pulse of westerly wind is replaced by a stronger wind feature at a different latitude. The jet speed spectrum also has a peak at 365 days and another clear peak at 182.5 days, showing that the second harmonic is also required to describe the seasonal cycle in this case.

Osprey and Ambaum (2011) recently showed that the spectra of the Northern Annular Mode exhibits an exponential power spectra at periods shorter than 36 days, indicative of chaotic behaviour. Interestingly, the spectra shown in Figure 2 do not fit an exponential decay particularly well, an impression which is confirmed when the spectra are replotted with a linear $\mathrm{x}$ axis (not shown). This difference may arise from the nature of the jet diagnostics, in which wind maxima are sought in low-pass filtered data, or from the regional rather than hemispheric focus taken here.

The seasonal cycles themselves are shown in Figure 3. The general shape of these is as expected from the changes 
in temperature gradients, with the jet strengthening and moving equatorward during winter. The jet latitude cycle shows considerable lag with respect to the insolation, with the jet at its most equatorward in March-May rather than the conventional December-February winter season. The jet proceeds poleward during the summer and reaches its maximum latitude in September. Although the seasonal cycle emerges clearly in both this and the spectral analysis, it is in fact rather small, with the jet moving only around $5^{\circ}$ over the year. This variation is much smaller than the month-to-month variability, as shown by the vertical bars. For example, there are clearly many examples of winter months when the jet position has been further poleward than the average summer monthly position. This is consistent with the results of Franzke and Woollings (2011) where it was shown that only $3 \%$ of the variance of jet latitude occurs on timescales longer than a year.

The seasonal cycle of jet speed is more pronounced compared to the variability, although there are clearly still many winter months with jet speeds as weak as those typically seen in summer. The contribution of the semiannual period acts to add an asymmetry to the cycle, so that the jet rapidly weakens from its strongest in January to its weakest in May. It is not clear what physical processes lie behind this asymmetry.

The seasonal cycles of three 45-year subsets are also shown in this figure. These show that there can be considerable differences in the shape of the cycles between different periods, which can give different impressions of the symmetry for example. The notion of convergence is somewhat ill-founded in the presence of time-varying forcings, but this does suggest that even periods this long may not be definitive for the purpose of assessing the climatology of a numerical model for example.

\section{Jet index distributions}

Woollings et al. (2010) showed that in the ERA-40 reanalysis the distribution of the daily jet latitude index in North Atlantic winter exhibits a trimodal structure which could reflect the existence of distinct flow regimes (see also Franzke et al. (2011); Hannachi et al. (2012)). We present the same analysis for the Twentieth Century reanalysis data in Figure 4. In winter this shows a very similar trimodal structure to that seen in ERA-40. This supports the robustness of this distribution and shows that it is not an accident of the recent few decades. The other seasons show more structure in the distributions than seen in ERA40 , with some evidence of preferred flow regimes in spring and autumn in particular. Interestingly, the locations of these seem very similar in the different seasons, which suggests that the occurrence of preferred jet positions may be related to the location of physical features such as orography, coastlines or ocean currents.

Figure 5a shows the variability of the wintertime distribution between individual 20-year periods. In general, each of the three peaks in the distribution is represented at the same location in each of the periods, although the southern jet position was particularly rare in both the $1870 \mathrm{~s} / 80 \mathrm{~s}$ and $1990 \mathrm{~s} / 2000 \mathrm{~s}$. This figure gives the impression that decadal jet variability is associated with low-frequency changes in the occurrence of each of the three fixed jet positions. Such decadal-scale variations in the occurrence frequencies of the three distinct jet regime states have been reported in the shorter ERA-40 record by (Franzke et al. 2011). This impression is further confirmed by Figure $5 \mathrm{~b}$ which shows the standard deviation of the PDFs from the individual periods (literally the standard deviation of the 7 curves in Figure 5a). This shows that the decadal variations in the jet latitude PDF are largest at the latitudes of the peaks in the PDF, so that it is indeed changes in the occurrence of the three jet positions which are dominating the decadal variability.

In contrast, the distributions of jet speed are all unimodal (not shown). However, the distributions are positively skewed for all months of the year, with skewness values between 0.1 and 0.7 (Czuchnicki 2011). This could reflect the nonlinearities of boundary layer dynamics, as described by Monahan (2006). In addition, some of the skewness in 
the seasonal distributions for the transition seasons of MAM and SON can be attributed to an artefact of the seasonal cycle. For example, the jet speed distribution in MAM has a strong positive skew because the distribution in March is shifted towards stronger winds than in April and May, while the distributions for each of the individual months all have weaker skewness.

\section{Long-term jet variability}

As suggested by Figure 5, the jet latitude index displays considerable variability on interannual and decadal timescales. Figure 6 shows time series of the seasonal mean jet latitude, formed by simply averaging the daily jet latitude values over the corresponding season. Firstly we note that the spread between ensemble members is relatively small, in particular in winter. This gives confidence that the observational coverage is dense enough to constrain the reanalysis even in the late 19th century. The ensemble spread is larger in the other seasons, which may reflect the weaker nature of large scale pressure patterns so that surface pressure observations provide weaker constraints on the general circulation. Despite this spread there is clearly much agreement across the ensemble on many of the jet variations in the early period.

In all seasons the range of interannual variability is of order $5-10^{\circ}$, so it is as large as the mean seasonal cycle. Strong multi-year variability is evident in all seasons, although weakest in autumn. Franzke and Woollings (2011) noted a positive trend in jet latitude over the ERA40 period in an Empirical Mode Decomposition analysis which used data from all seasons. In agreement with this, Figure 6 shows positive jet latitude trends since 1960 in all seasons except autumn. However, these trends do not appear remarkable in the context of the longer records, and the jet latitude in recent decades has not exceeded values seen in earlier periods. The strong winter NAO trend from the 1960 s to the 1990 s does not appear so striking in terms of jet latitude in this longer record.
The corresponding time series of jet speed are shown in Figure 7. These also show considerable levels of low frequency variability with several instances of consecutive decades of anomalous values. As seen for jet latitude, the speed can exhibit year-to-year variations as large as the seasonal cycle. In some seasons the jet speed seems to have changed considerably over the first few decades of the reanalysis. While the ensemble spread again appears modest these changes could reflect the drifting of the model back towards its own climatology in periods of sparse observations, as described in section 3. As for jet latitude, the recent few decades are not unusual in the long term context. In particular, there is no evidence by this measure of a weakening in the summertime westerlies as suggested to have arisen from enhanced Arctic warming by Francis and Vavrus (2012).

Trend analyses have been performed on the indices using the Empirical Mode Decomposition method as in Franzke and Woollings (2011). This method defines the trend as the residual after all empirical oscillations have been removed from the time series. Note that the method is applied to the continuous daily time series and so cannot distinguish between different seasons. This analysis finds that the jet latitude exhibits a positive trend in every ensemble member which is significant at the $97.5 \%$ level. The mean poleward shift is $2.8^{\circ}$ over the whole period, or about $0.2^{\circ}$ per decade. This is small compared to the interannual/decadal variability but still statistically significant. It is not clear whether this trend reflects a response to external forcing or not. In contrast, none of the ensemble members shows a significant trend in jet speed.

As in the ERA-40 analysis of Woollings and Blackburn (2012), the time series of jet latitude and speed are not significantly correlated. While patterns of variability such as the NAO combine variations in jet latitude and speed it seems that their variability is quite different and so they may be influenced by different factors. This is also supported by the clearly different seasonal cycles shown in section 4 . 
Power spectra of the seasonal time series are shown in Figures 8 and 9. In general the spectra are flat and so are similar to that of a white noise process. Given the variability in regime occurrence shown in Figure 5 it is interesting that the wintertime jet latitude spectra in Figure 8 does not show strong variability at very low frequencies. In particular for jet speed, however, some of the spectra do show high power at the lowest frequencies, consistent with the impression of high multi-decadal variability in the time series of Figure 7. A key question for future research is to what extent decadal jet variability is consistent with internal tropospheric variability and how large a role external forcing agents play. However, one practical consequence of these results is that for the purpose of model evaluation there may be some sensitivity of the jet speed to the choice of observational period used.

\section{Changes in intraseasonal variability}

Häkkinen et al. (2011) recently analysed wintertime atmospheric blocking in the Twentieth Century reanalysis and suggested that the occurence of Euro-Atlantic blocking has varied on decadal timescales in phase with the Atlantic Multidecadal Oscillation (AMO). A novel feature of their analysis is that blocking events all across the Atlantic and Europe are combined into one time series of occurrence. This process combines, for example, periods when the jet is displaced south of a Greenland block (Scherrer et al. 2006; Croci-Maspoli et al. 2007; Woollings et al. 2008) with periods when the jet is displaced north of an Iberian block (Woollings et al. 2011; Davini et al. 2012). In this way a period of enhanced blocking in their analysis could correspond to a period of strong intraseasonal variability in jet latitude.

Motivated by this, we show in Figure 10 the time series of intraseasonal jet latitude variability, defined as the standard deviation of the daily jet latitude values in each individual season. As anticipated, this time series shows considerable long-term changes in intraseasonal variability, with some similarity to the variations in blocking described by Hakkinen et al. (their fig 2b). The decadal changes are larger than the ensemble spread for much of the time series (see also Figure 1b which shows the ensemble spread of the unfiltered series). This suggests relatively low observational uncertainty in this variability, a factor which was not addressed in Hakkinen et al. There is also some similarity to the AMO variations, which are shown in red, although the two diverge in particular in the 1870s and 1960s. It is of course possible that the discrepancy in the 1870s arises due to a lack of observations as described in section 3, especially given the larger ensemble spread in that period. Ideally, of course, even longer records should be used to evaluate the correlation of variations on this timescale.

Hakkinen et al approach their blocking-AMO relation largely from the perspective of the influence of the atmosphere on the ocean, via changes in the patterns of wind stress curl. From this perspective the results presented here may be consistent with the suggestion of Czaja (2009) that the strong variability in Atlantic jet latitude is crucial in the maintenance of the Atlantic Meridional Overturning Circulation (AMOC). Periods of anomalously high variability in jet latitude could then lead to enhanced transport of salinity to high latitudes which could act to enhance the AMOC and so influence the AMO. It is not clear, however, if this process can act on the timescale needed to explain the relation suggested by Figure 10.

An obvious and important question concerns the origin of the decadal variations in jet variability themselves. Could these have arisen from climate noise (Feldstein 2000; Czaja et al. 2003; Franzke 2009) or are they reliant on some form of external forcing such as the variations in the AMO itself? To investigate this issue we compare the observations to a simple statistical model representing jet latitude fluctuations. In this case we use a Autoregressive Moving Average (ARMA) model for the winter season (December through February) as described by Franzke and Woollings (2011). The best fit ARMA model has been determined separately for the jet latitude in each ensemble member. These ARMA models are then integrated to give 1000 
synthetic time series for each ensemble member from which time series of intraseasonal variability can be derived. The synthetic time series allow us to compute seasonal standard deviations of the fluctuations for each ensemble member separately. We then use the 5th and 95th percentiles of the standard deviations to gauge if the observed interannual jet latitude fluctuations are consistent with the fluctuations generated by the ARMA model. The orders of the ARMA model varies from about 3 to 10 amongst the ensemble members suggesting that individual ensemble members exhibt quite different temporal dynamics. However, our Monte Carlo approach takes account of this uncertainty.

Figure 10 shows the results of this analysis. In order to focus on decadal scale variability the time series has been smoothed with a 11 point Gaussian filter. The observed decadal variations in intraseasonal variability (solid line) is almost always within the bounds of the decadal variability seen in the ARMA ensemble (dashed lines), showing that this level of decadal variability can arise without the need for memory in the system beyond a few days. This memory as measured by the order of the ARMA process is not longer than the typical life cycle of teleconnection patterns (Feldstein 2000, Franzke and Feldstein 2005) or blocking (Tyrlis and Hoskins 2008). Though we cannot prove that there were no external influences our Monte Carlo results suggest that external forcing is not required to explain this variability. If the atmospheric variability does influence the ocean circulation, as proposed in Hakkinen et al, then this influence could comprise the ocean acting as an integrator of stochastic atmospheric forcing containing all timescales, as in Hasselmann (1976) and Frankignoul and Hasselmann (1977). Our results might suggest that the atmosphere is driving the ocean (Czaja 2009) but do not provide evidence that the ocean exerts an influence on the jet stream in the North Atlantic region.

\section{Conclusions}

We have used the Twentieth Century reanalysis to derive a long record of variations in the latitude and speed of the North Atlantic eddy-driven jet. This reanalysis product allows some estimation of the circulation uncertainty due to the provision of multiple ensemble members. In the North Atlantic the jet characteristics are well constrained by the available observations over the 20th century, and show reasonable agreement in the late 19th century, in particular in winter. This is often not the case in other regions. Specific conclusions derived from this record are listed below.

- Both the latitude and speed of the jet have shown considerable multi-year/decadal variability over the 20th century in all seasons. The changes over the recent few decades do not appear unusual compared to previous variations, and recent events are not unprecedented in the historical record.

- The seasonal cycle of jet latitude in particular is quite modest compared to the large amount of variability on monthly/seasonal timescales.

- The latitude and speed of the jet are not correlated in their variability and they exhibit quite different seasonal cycles. This suggests that they may have quite different dynamical influences.

- The trimodal distribution of jet latitude in winter is a robust feature of this longer reanalysis, and there is also evidence of similar preferred jet positions in the transition seasons.

- While general features of the jet statistics are robust, there can be significant differences between periods as long as 40 years in features such as the mean jet speed, the detailed shape of the jet latitude PDF and the apparent structure in the seasonal cycle. This high level of variability suggests long time periods may be needed for precise evaluations of climate models.

- The decadal variability in jet latitude is associated with changes in the occurrence of the different jet positions.

- The level of wintertime intraseasonal variability in jet latitude has itself varied on decadal timescales. These variations show some similarity to the AMO and are 
not inconsistent with the variability exhibited by a simple statistical noise model with memory of only a few days.

\section{Acknowledgement}

Support for the Twentieth Century Reanalysis Project dataset is provided by the U.S. Department of Energy, Office of Science Innovative and Novel Computational Impact on Theory and Experiment (DOE INCITE) program, and Office of Biological and Environmental Research (BER), and by the National Oceanic and Atmospheric Administration Climate Program Office.

\section{References}

Archer, C. L. and Caldeira, K. (2008). Historical trends in the jet streams. Geophys. Res. Lett., 35, 108803.

Cattiaux, J., Vautard, R., Cassou, C., Yiou, P., Masson-Delmotte, V., and Codron, F. (2010). Winter 2010 in Europe: A cold extreme in a warming climate. Geophys. Res. Lett., 37, 20704.

Cohen, J. and Barlow, M. (2005). The NAO, the AO, and Global Warming: How Closely Related? Journal of Climate, 18, 4498-4513.

Compo, G. P., Whitaker, J. S., Sardeshmukh, P. D., Matsui, N., Allan, R. J., Yin, X., Gleason, B. E., Vose, R. S., Rutledge, G., Bessemoulin, P., Brönnimann, S., Brunet, M., Crouthamel, R. I., Grant, A. N., Groisman, P. Y., Jones, P. D., Kruk, M. C., Kruger, A. C., Marshall, G. J., Maugeri, M., Mok, H. Y., Nordli, Ø., Ross, T. F., Trigo, R. M., Wang, X. L., Woodruff, S. D., and Worley, S. J. (2011). The Twentieth Century Reanalysis Project. Q. J. R. Meteorol. Soc., 137, $1-28$.

Croci-Maspoli, M., Schwierz, C., and Davies, H. (2007). Atmospheric blocking - Space-time links to the NAO and PNA. Climate Dynam., 29, 713-725.

Czaja, A. (2009). Atmospheric Control on the Thermohaline Circulation. Journal of Physical Oceanography, 39, 234.

Czaja, A., Robertson, A. W., and Huck, T. (2003). The role of atlantic ocean-atmosphere coupling in affecting north atlantic oscillation variability. In J. Hurrell, Y. Kushnir, G. Ottersen, and M. Visbeck, editors, The North Atlantic Oscillation: Climatic Significance and Environmental Impact, volume 134 of Geophysical Monographs, pages 147-172. American Geophysical Union.

Czuchnicki, C. (2011). Seasonality of the eddy-driven jet stream variability and change. Master's thesis, Dept. of Meteorology, University of Reading.
Davini, P., Cagnazzo, C., Gualdi, S., and Navarra, A. (2012). Bidimensional diagnostics, variability and trends of northern hemisphere blocking. J. Climate. Accepted.

Feldstein, S. B. (2000). The timescale, power spectra, and climate noise properties of teleconnection patterns. J. Climate, 13, 4430-4440.

Francis, J. A. and Vavrus, S. J. (2012). Evidence linking arctic amplification to extreme weather in mid-latitudes. Geophys. Res. Lett., 39, L06801.

Frankignoul, C. and Hasselmann, K. (1977). Stochastic climate models, part II. Application to sea-surface temperature anomalies and thermocline variability. Tellus, 29, 289.

Franzke, C. (2009). Multi-scale analysis of teleconnection indices: climate noise and nonlinear trend analysis. Nonlinear Proc. Geoph., 16, 65-76.

Franzke, C. and Woollings, T. (2011). On the persistence and predictability properties of north atlantic climate variability. $J$. Climate, 24, 466-472.

Franzke, C., Woollings, T., and Martius, O. (2011). Persistent Circulation Regimes and Preferred Regime Transitions in the North Atlantic. J. Atmos. Sci., 68, 2809-2825.

Häkkinen, S., Rhines, P. B., and Worthen, D. L. (2011). Atmospheric Blocking and Atlantic Multidecadal Ocean Variability. Science, 334, $655-$.

Hannachi, A., Woollings, T., and Fraedrich, K. (2012). The North Atlantic jet stream: a look at preferred positions, paths and transitions. Q. J. R. Meteorol. Soc. online.

Hasselmann, K. (1976). Stochastic climate models, Part I, Theory. Tellus, 28, 473 .

Hurrell, J. W. (1995). Decadal trends in the North Atlantic Oscillation: Regional temperatures and precipitation. Science, 269, 676-679.

Hurrell, J. W. and Deser, C. (2009). North Atlantic climate variability: The role of the North Atlantic Oscillation. Journal of Marine Systems, 78, 28-41.

Jones, P. D., Jonsson, T., and Wheeler, D. (1997). Extension to the North Atlantic oscillation using early instrumental pressure observations from Gibraltar and south-west Iceland. International Journal of Climatology, 17, 1433-1450.

Luterbacher, J., Xoplaki, E., Dietrich, D., Jones, P. D., Davies, T. D., Portis, D., Gonzalez-Rouco, J. F., von Storch, H., Gyalistras, D., Casty, C., and Wanner, H. (2001). Extending North Atlantic oscillation reconstructions back to 1500. Atmospheric Science Letters, 2, 114-124.

Monahan, A. H. (2006). The Probability Distribution of Sea Surface Wind Speeds. Part I: Theory and SeaWinds Observations. J. Climate, 19, 497-519. 
Osprey, S. M. and Ambaum, M. H. P. (2011). Evidence for the chaotic origin of Northern Annular Mode variability. Geophys. Res. Lett., 38, 15702 .

Pinto, J. G. and Raible, C. C. (2012). Past and recent changes in the North Atlantic oscillation. WIREs Clim Change, 3, 79-90.

Scherrer, S. C., Croci-Maspoli, M., Schwierz, C., and Appenzeller, C. (2006). Two-dimensional indices of atmospheric blocking and their statistical relationship with winter climate patterns in the EuroAtlantic region. International Journal of Climatology, 26, 233-249.

Strong, C. and Davis, R. E. (2007). Winter jet stream trends over the northern hemisphere. Q. J. R. Meteorol. Soc., 133, 2109-2115.

Strong, C. and Davis, R. E. (2008). Variability in the Position and Strength of Winter Jet Stream Cores Related to Northern Hemisphere Teleconnections. Journal of Climate, 21, 584-592.

Thompson, D. W. J. and Wallace, J. M. (2001). Regional climate impacts of the Northern Hemisphere Annular Mode. Science, 293, 85-89.

Tyrlis, E. and Hoskins, B. J. (2008). Aspects of Northern Hemisphere atmospheric blocking climatology. J. Atmos. Sci., 65, 1638-1652.

Woollings, T. and Blackburn, M. (2012). The North Atlantic jet stream under climate change, as described by the NAO and EA patterns. $J$. Climate, 25, 886-902.

Woollings, T., Hannachi, A., and Hoskins, B. (2010). Variability of the North Atlantic eddy-driven jet stream. Q. J. R. Meteorol. Soc., 649, $856-868$.

Woollings, T., Pinto, J. G., and Santos, J. A. (2011). Dynamical Evolution of North Atlantic Ridges and Poleward Jet Stream Displacements. J. Atmos. Sci., 68, 954-963.

Woollings, T. J., Hoskins, B. J., Blackburn, M., and Berrisford, P. (2008). A new Rossby wave-breaking interpretation of the North Atlantic Oscillation. J. Atmos. Sci., 65, 609-626. 

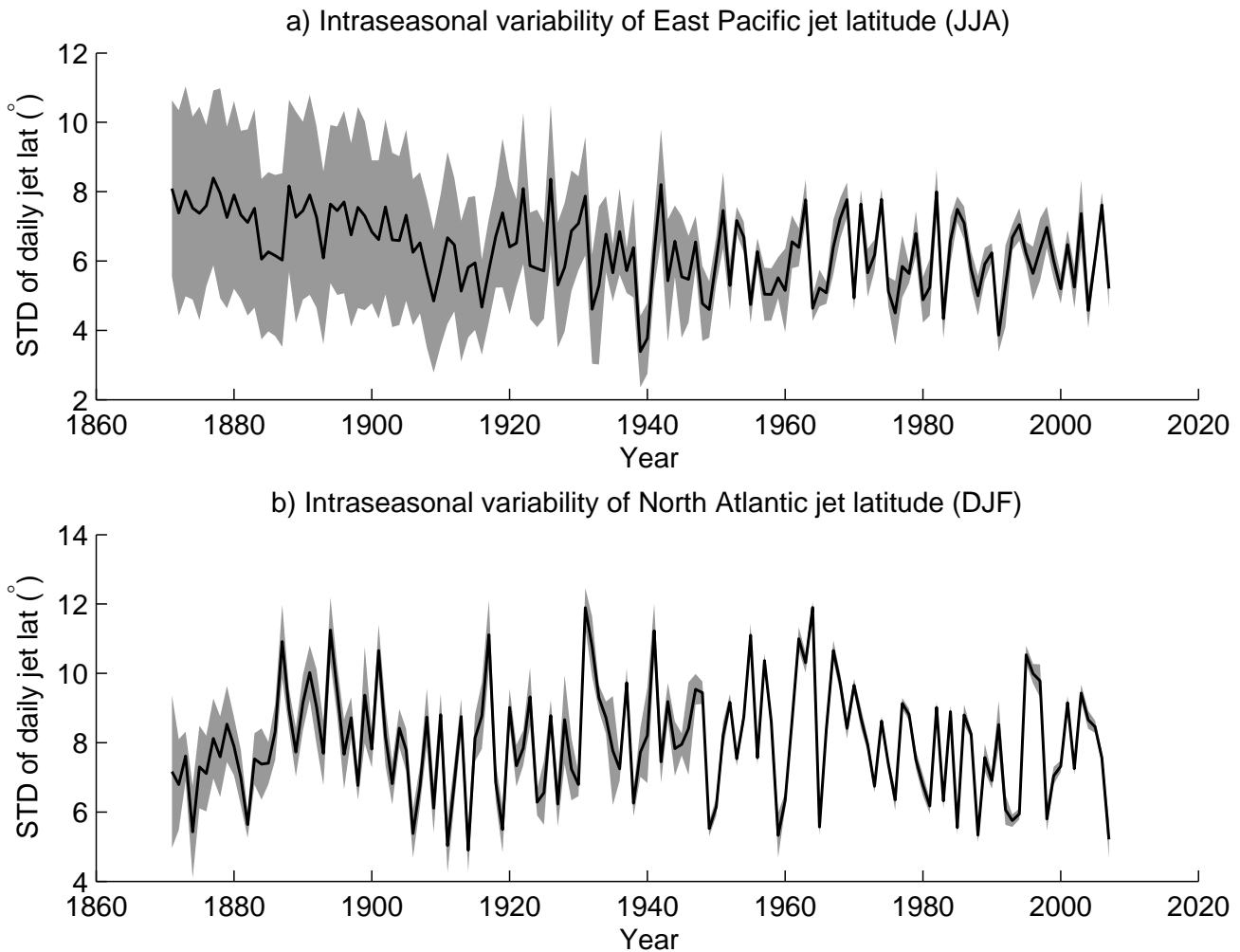

Figure 1. Time series of the intraseasonal variability of the jet speed. This series is formed by taking the standard deviation of the 90 days in each individual season. Shading shows the \pm 2 standard deviation range across the ensemble. a) Eastern North Pacific (200-240 ${ }^{\circ} \mathrm{E}$ ) JJA; b) North Atlantic $\left(0-60^{\circ} \mathrm{W}\right) \mathrm{DJF}$. 
a) Jet latitude

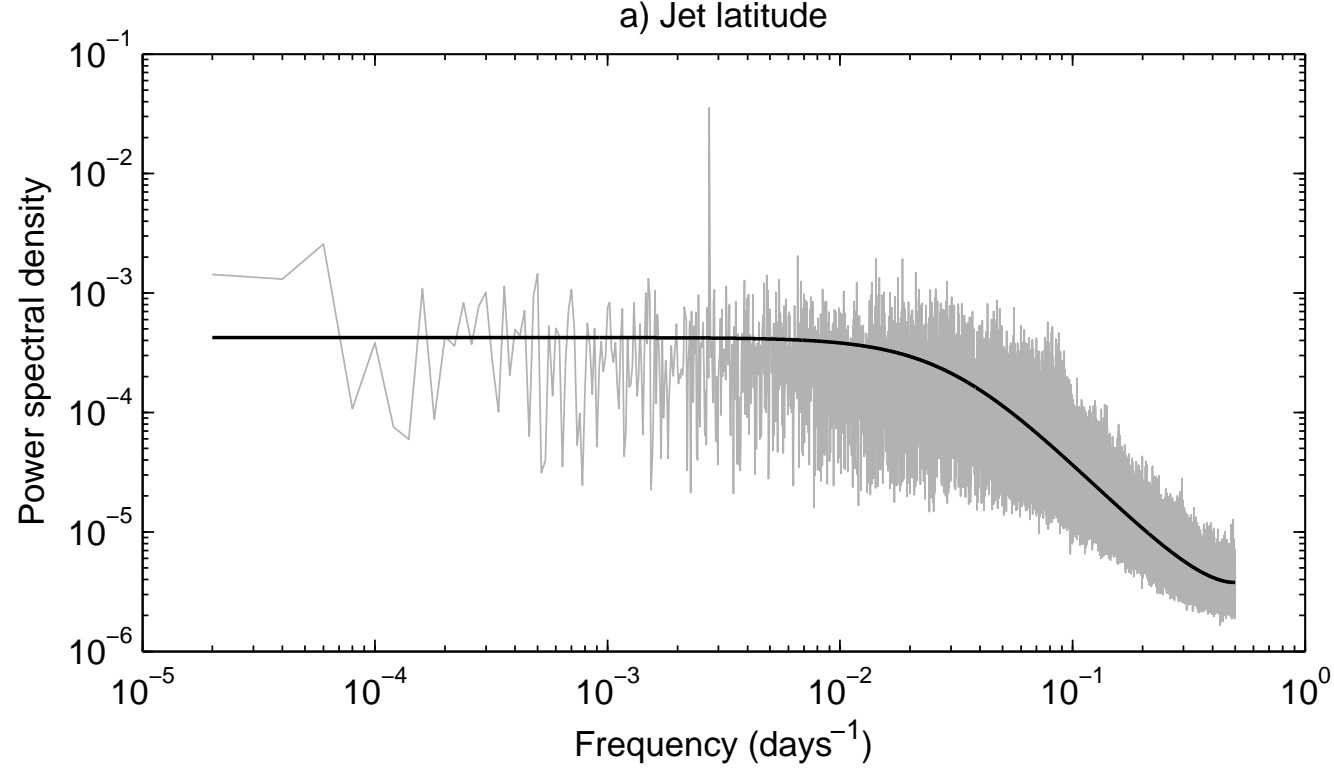

b) Jet speed

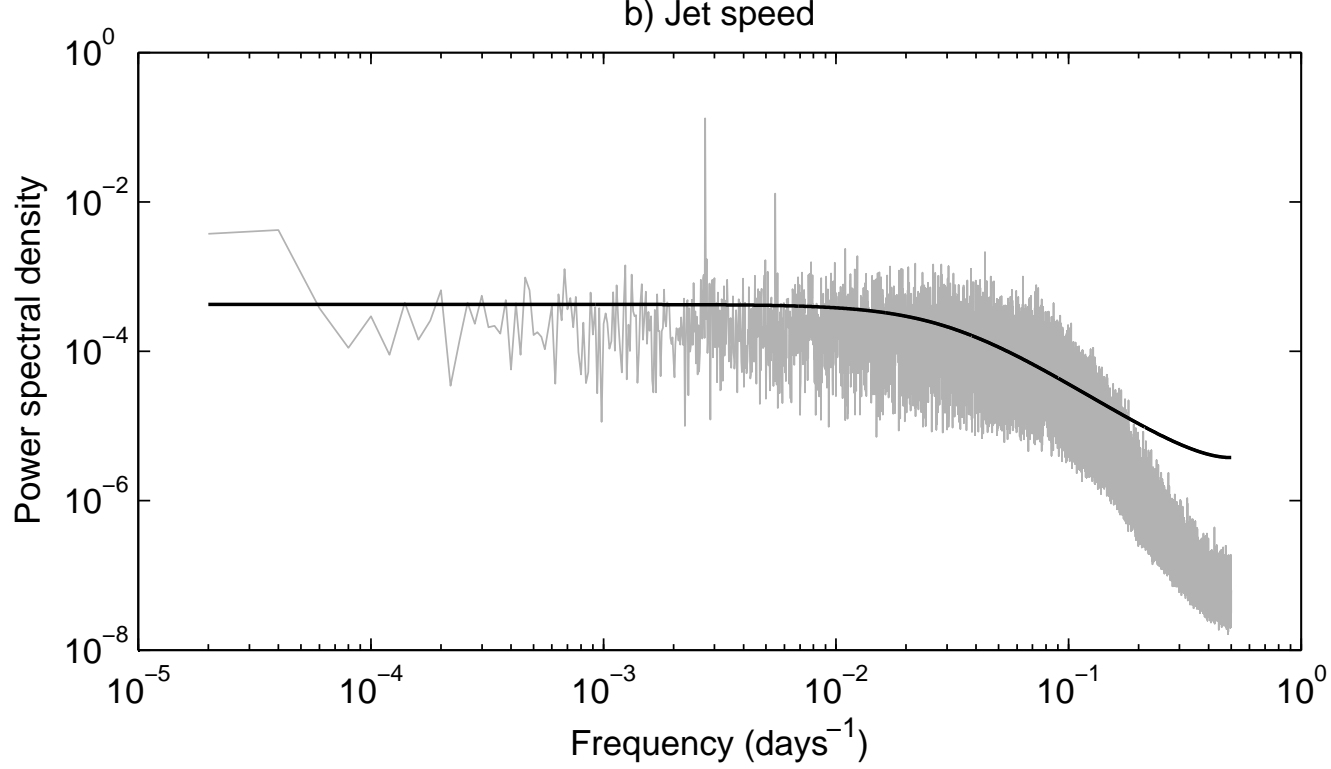

Figure 2. Daily spectra of North Atlantic jet latitude (a) and speed (b).

The black lines indicate the theoretical spectra of the corresponding AR1

process derived from the variance and lag- 1 autocorrelation of the series.

Note that leap days have been neglected for this figure. 
a) Seasonal cycle of North Atlantic jet latitude

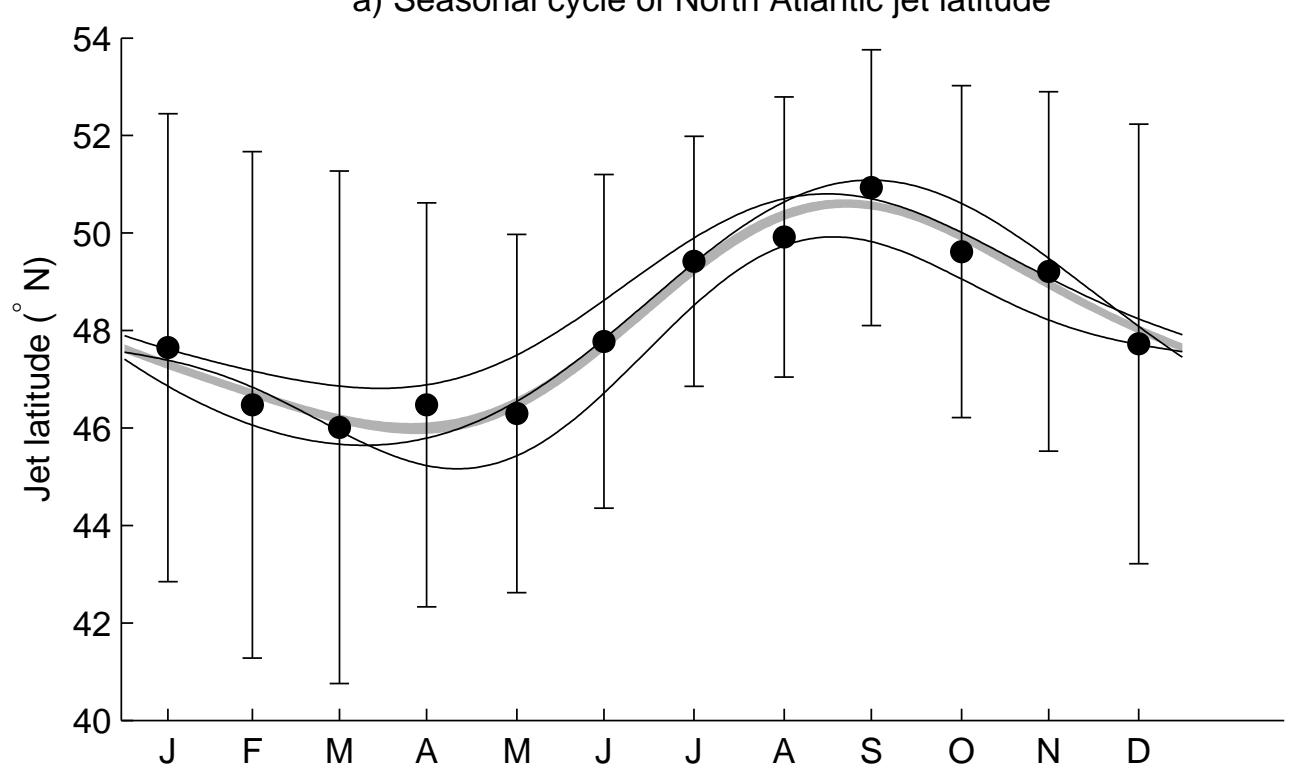

b) Seasonal cycle of North Atlantic jet speed

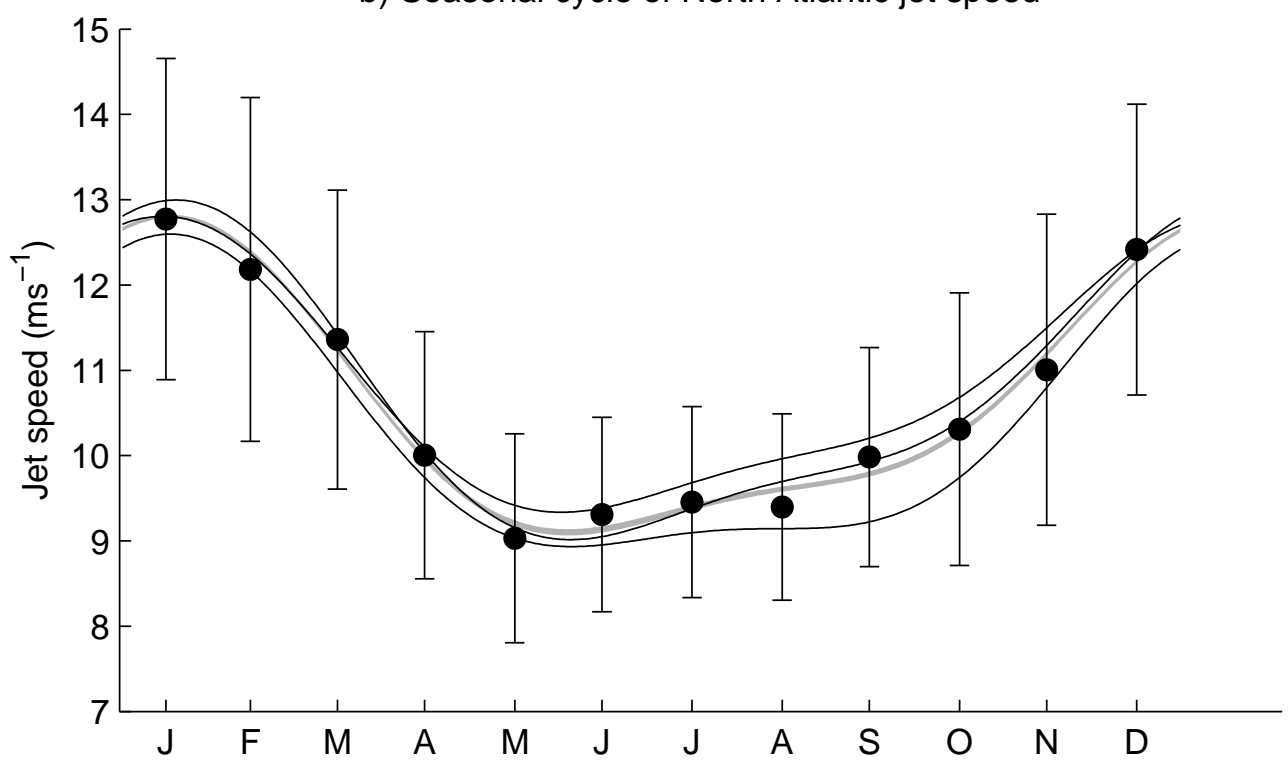

Figure 3. Seasonal cycles of the jet latitude (a) and speed (b). The seasonal cycles are derived by averaging the jet indices over all complete years of the reanalysis. The daily-resolved cycles are shown by the grey shading which indicates the \pm 2 standard deviation range across the 56 ensemble members. The individual black lines show the daily cycles exhibited by subsamples of three non-overlapping 45 year periods. Black dots mark the monthly averages of the ensemble mean data, with bars indicating ranges of \pm 1 standard deviation across the $136 / 7$ months of the record. 

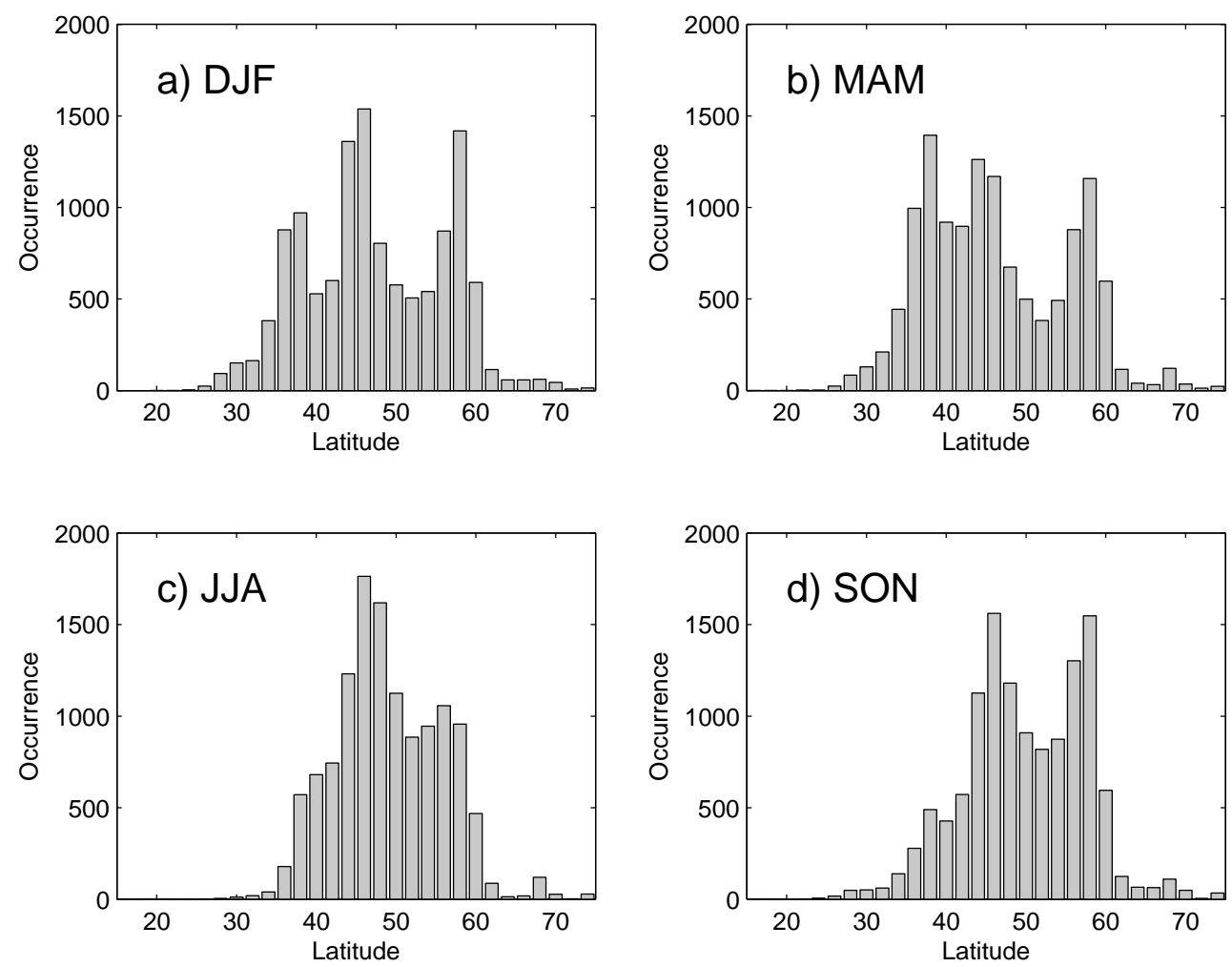

Figure 4. Ensemble mean distributions of daily jet latitude in the four seasons. The standard deviation between the ensemble members is typically only 20-30 occurrences on the scale used here, so is not shown. 
a) 20-winter jet latitude PDFs

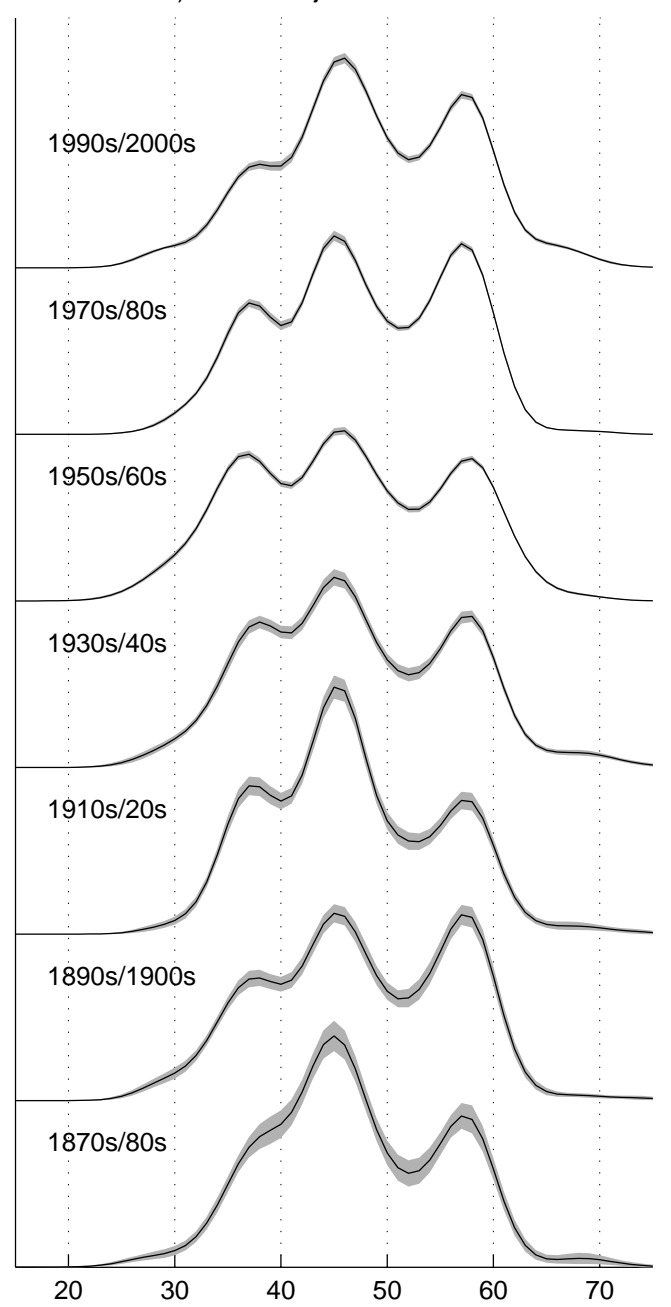

b) STD between 20-winter periods

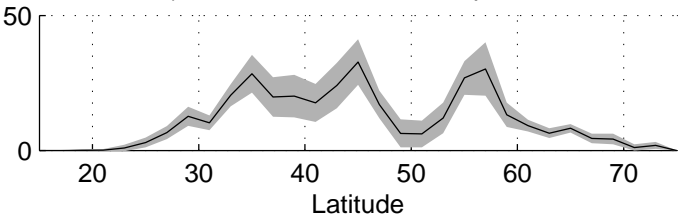

Figure 5. a) PDFs of wintertime jet latitude constructed for 20-year periods by applying a kernel smoothing to the distributions as in Woollings et al. (2010). b) Standard deviation of the 7 PDFs in panel a). In both panels, shading indicates the \pm 2 standard deviation range across the ensemble members. 
a) Jet latitude: DJF

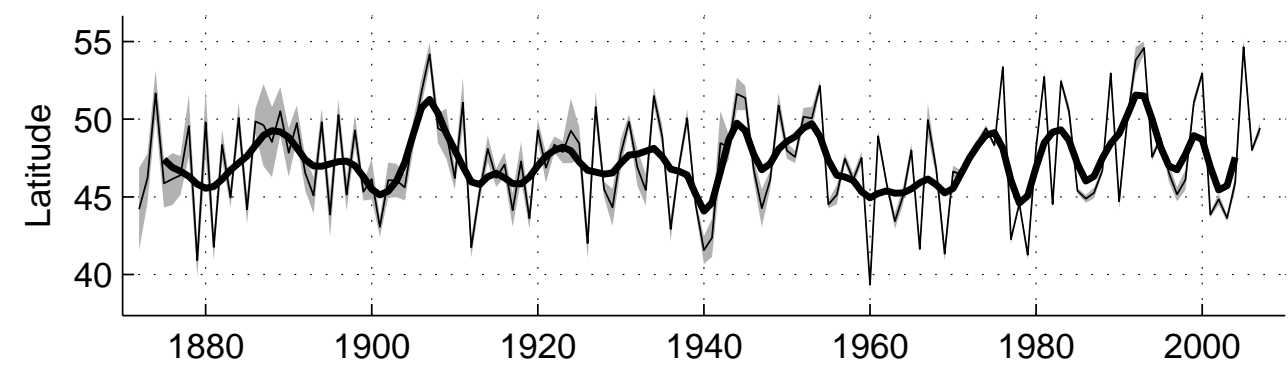

b) Jet latitude: MAM

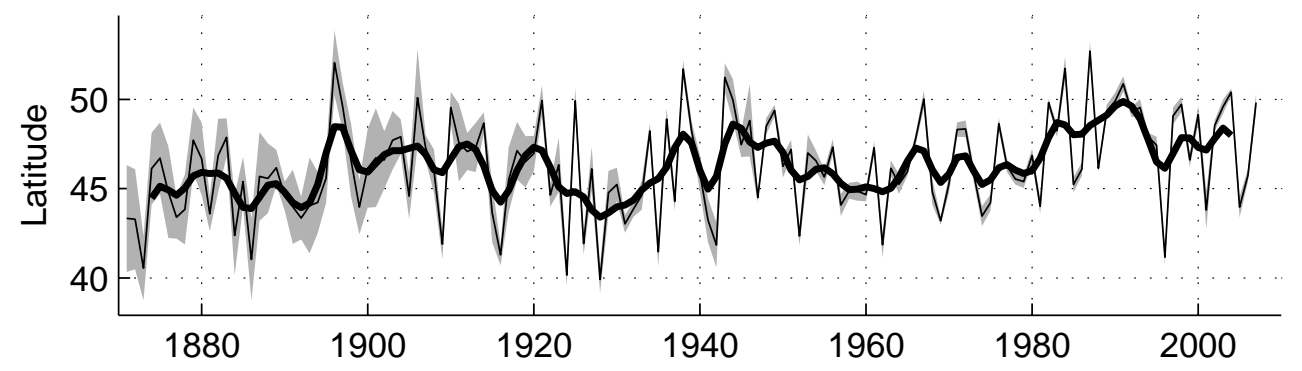

c) Jet latitude: JJA

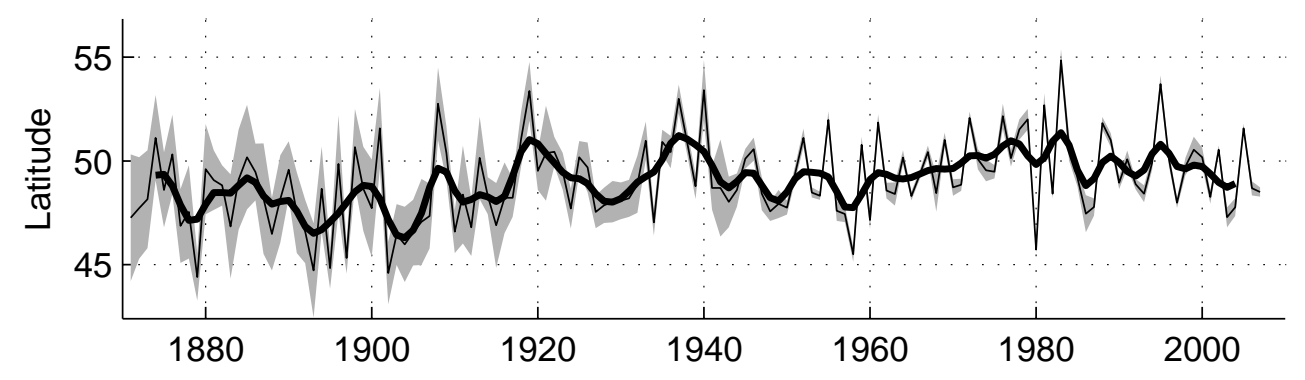

d) Jet latitude: SON

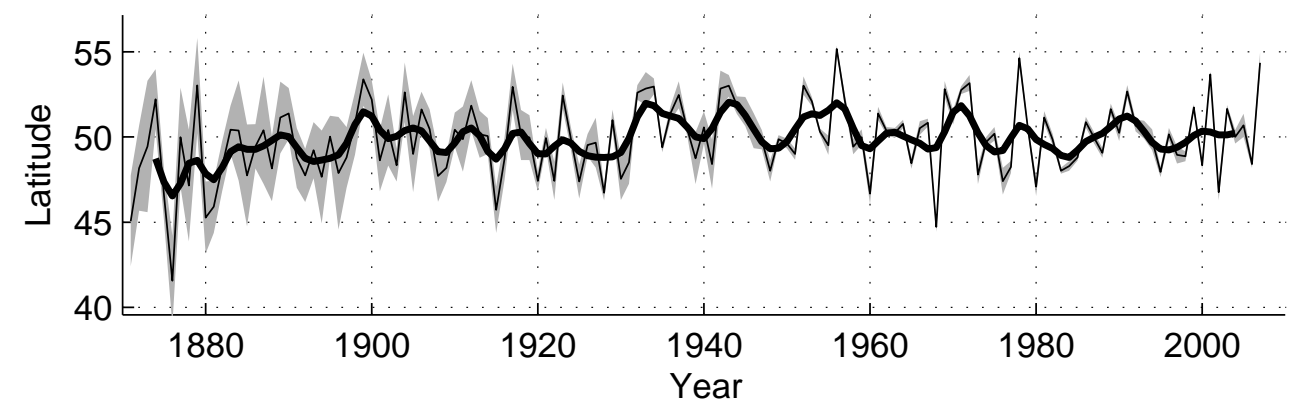

Figure 6. Time series of seasonal mean jet latitude, with the \pm 2 standard deviation range across the ensemble shaded. The thick lines show versions which have been smoothed with a 7 point binomial filter. 
a) Jet speed: DJF

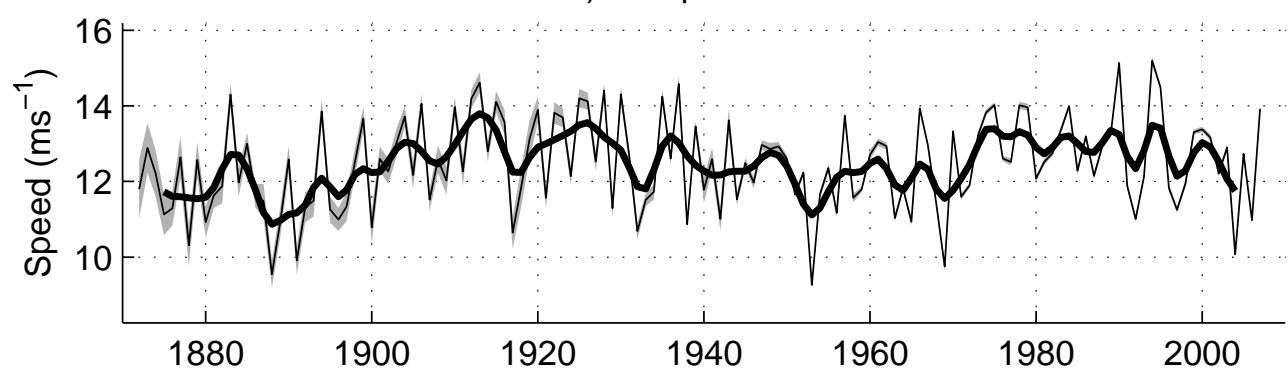

b) Jet speed: MAM

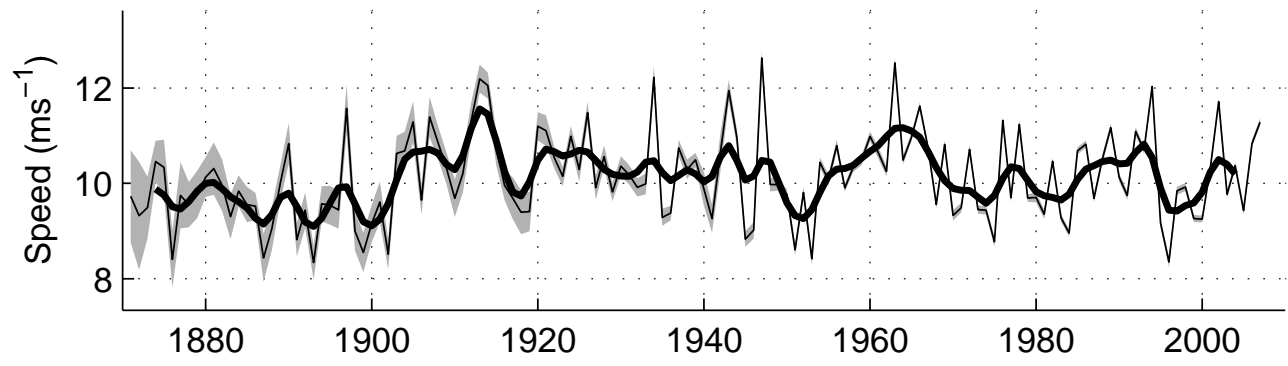

c) Jet speed: JJA
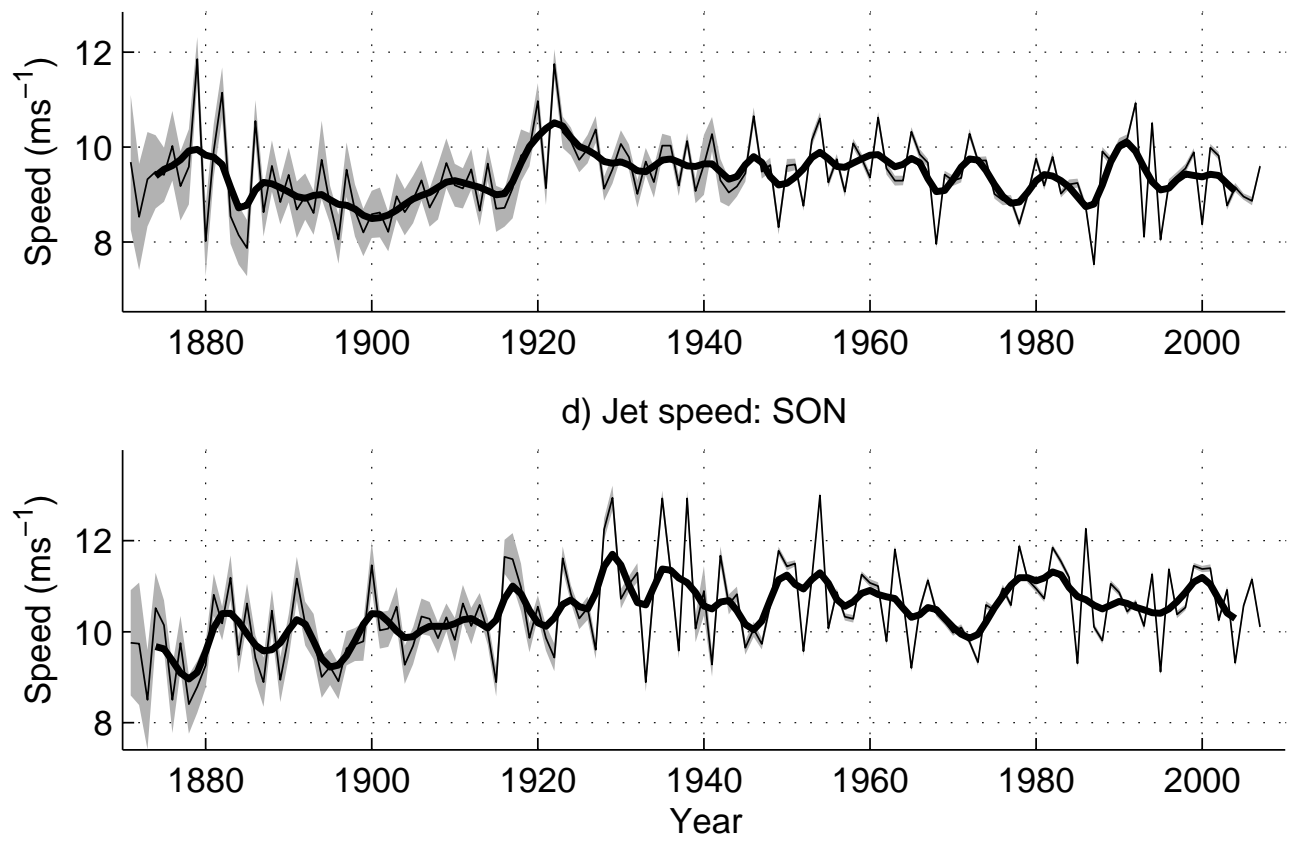

Figure 7. Time series of seasonal mean jet speed, with the \pm 2 standard deviation range across the ensemble shaded. The thick lines show versions which have been smoothed with a 7 point binomial filter. 
a) Jet latitude: DJF

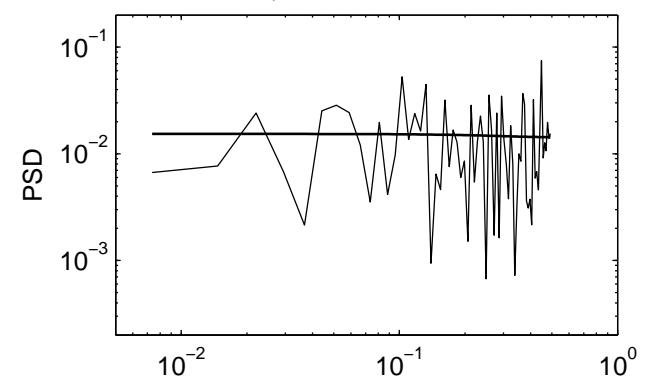

c) Jet latitude: JJA

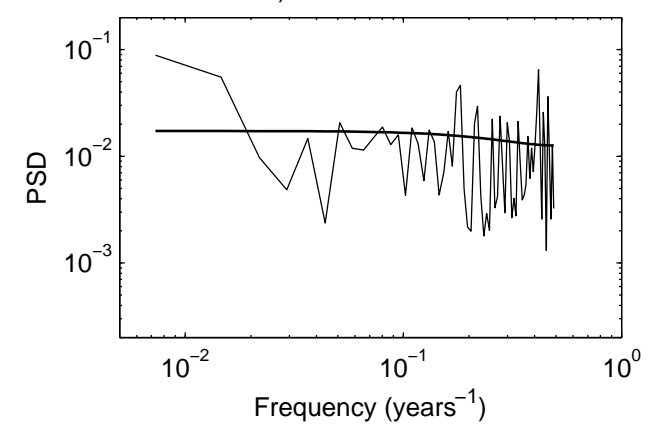

b) Jet latitude: MAM

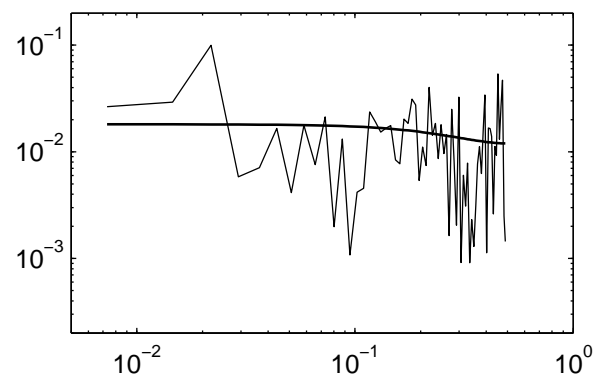

d) Jet latitude: SON

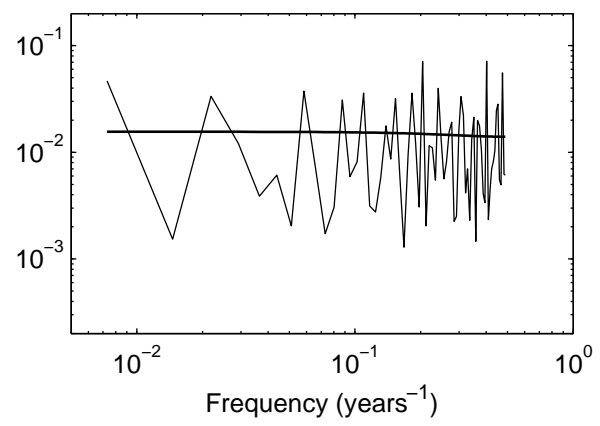

Figure 8. Power spectra of the seasonal-mean jet latitude series shown in Figure 6. The spectra are computed for each ensemble member and the ensemble mean is plotted here. The ensemble mean of the theoretical spectra of the associated AR1 processes is also plotted. 
a) Jet speed: DJF

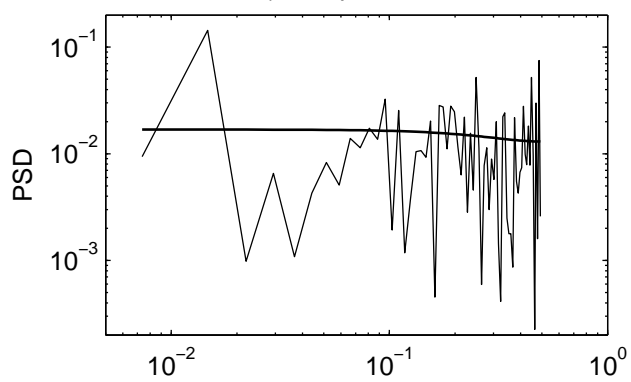

c) Jet speed: JJA

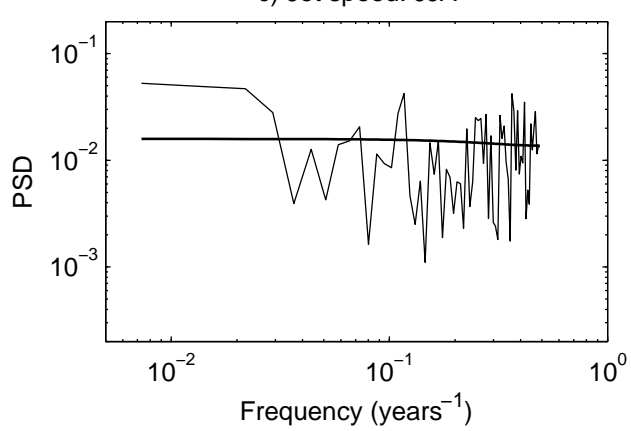

b) Jet speed: MAM

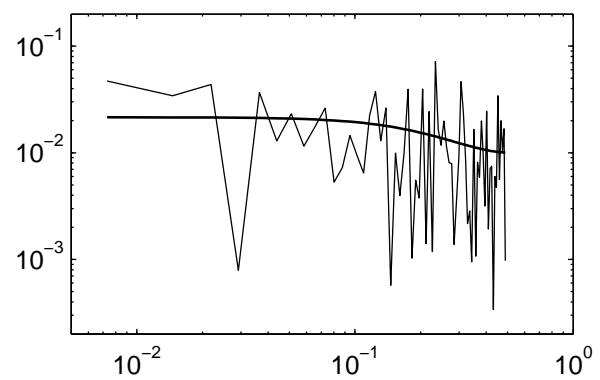

d) Jet speed: SON

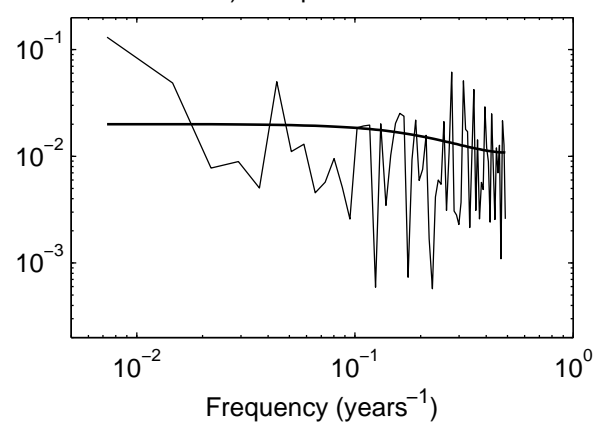

Figure 9. Power spectra of the seasonal-mean jet speed series shown in Figure 7. The spectra are computed for each ensemble member and the ensemble mean is plotted here. The ensemble mean of the theoretical spectra of the associated AR1 processes is also plotted. 


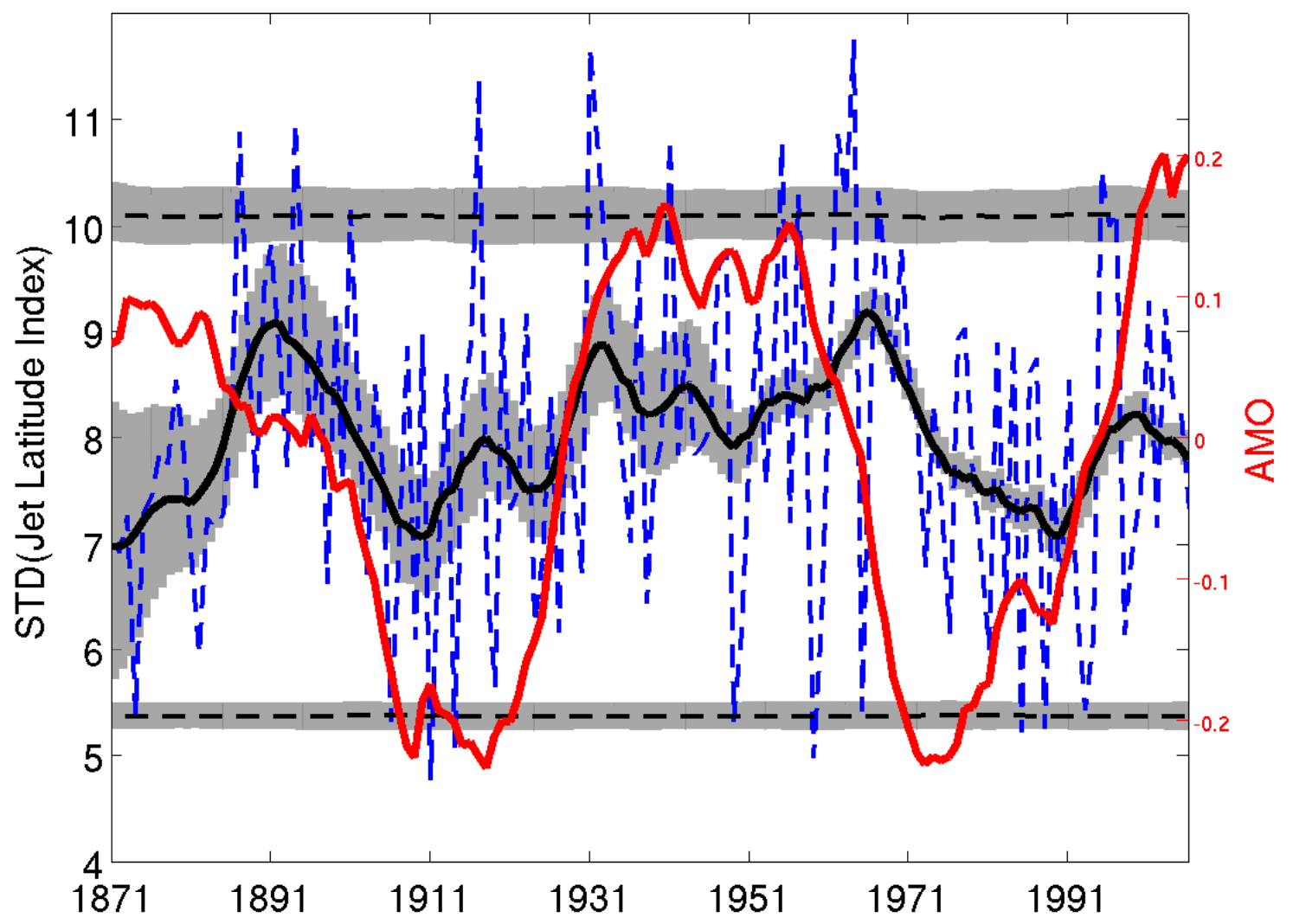

Figure 10. Time series of the standard deviation of daily jet latitude within each 90-day winter season. The blue dashed line indicates the ensemble mean. The black line shows the standard deviation of daily jet latitude smoothed with an 11 point Gaussian filter to isolate the decadal variations, with the 5th and 95th percentile range across the ensemble shown by shading. Dashed lines and associated shading mark the ensemble mean and the 5th and 95th percentile range of the 11-year smoothed ARMA simulations. Also plotted in red is a smoothed version of the AMO index smoothed with a 11 point Gaussian filter. 